\title{
A LAW OF THE ITERATED LOGARITHM FOR STABLE SUMMANDS \\ BY
}

R. P. PAKSHIRAJAN AND R. VASUDEVA

\begin{abstract}
Let $X_{1}, X_{2}, \ldots$ be a sequence of independent indentically distributed stable random variables with parameters $\alpha(0<\alpha<2)$ and $\beta$ $(|\beta|<1)$. Let $S_{n}=\sum_{i=1}^{n} X_{i}$. Suppose that $\left(S_{1, n}\right)$ and $\left(S_{2, n}\right)$ are independent copies of the sequence $\left(S_{n}\right)$. In this paper we obtain the set of all limit points in the plane of the sequence
\end{abstract}

$$
\left\{\left|n^{-1 / \alpha}\left(S_{1, n}-a_{n}\right)\right|^{1 /(\log \log n)},\left|n^{-1 / \alpha}\left(S_{2, n}-a_{n}\right)\right|^{1 /(\log \log n)}\right\}
$$

where $\left(a_{n}\right)$ is zero if $\alpha \neq 1$ and is $(2 \beta n \log n) / \pi$ if $\alpha=1$.

Introduction. Let $X_{1}, X_{2}, \ldots$ be a sequence of independent and identically distributed random variables with characteristic function $g(t)$ given by

$$
g(t)=\exp \left\{-|t|^{\alpha}(1+i \beta(t /|t|) w(t, \alpha))\right\}, \quad 0<\alpha<2
$$

where

$$
w(t, \alpha)= \begin{cases}\tan (\pi \alpha / 2) & \text { if } \alpha \neq 1, \\ (2 \beta / \pi) n \log n & \text { if } \alpha=1\end{cases}
$$

Write $S_{n}=\sum_{i=1}^{n} X_{i}$. Denote by $\left(S_{1, n}\right)$ and $\left(S_{2, n}\right)$ two independent copies of the sequence $\left(S_{n}\right)$ and by $\left(T_{n}\right)$ and $\left(U_{n}\right)$ the sequences $\left\{n^{-1 / \alpha}\left(S_{1, n}-a_{n}\right)\right\}$ and $\left\{n^{-1 / \alpha}\left(S_{2, n}-a_{n}\right)\right\}$, respectively, where $a_{n}$ is zero if $\alpha \neq 1$ and is $(2 \beta n \log n) / \pi$ if $\alpha=1$. Throughout the paper let $\theta_{n}$ stand for $(\log \log n)^{-1}$.

When $\beta=0$ Chover [2] has established that $P\left(\lim \sup _{n \rightarrow \infty}\left|T_{n}\right|^{\theta_{n}}=e^{1 / \alpha}\right)=$ 1 and that almost every point in $\left[1, e^{1 / \alpha}\right]$ is a limit point of $\left|T_{n}\right|^{\theta_{n}}$. Chover raises the question whether for this sequence points in $[0,1)$ are also limit points. Using a result of Chung and Fuchs [3] he notes that when $\alpha>1$, zero is a limit point. The results obtained below include a complete answer to Chover's question. To be precise we show that the set of all the limit points of $\left|T_{n}\right|^{\theta_{n}}$ coincides with the following intervals in the various cases: $\left[e^{-1 /(1-\alpha)}\right.$, $\left.e^{1 / \alpha}\right]$ if $\alpha<1,|\beta|<1 ;\left[1, e^{1 / \alpha}\right]$ if $\alpha<1$ and $\beta= \pm 1$ and $\left[0, e^{1 / \alpha}\right]$ if $1<\alpha<2$. Independently of us J. L. Mijnheer [7] has also obtained the limit sets in the cases $\alpha<1, \beta= \pm 1$ and $1<\alpha<2$; in the case $\alpha<1,|\beta|<1$

Received by the editors January 11, 1975.

AMS (MOS) subject classifications (1970). Primary 60F15.

- American Mathematical Society 1977 
he states that he does not know whether $\left[e^{-1 /(1-\alpha)}, e^{1 / \alpha}\right]$ are all the limit points.

The object of this paper is to obtain the set $K$ of all the limit points of the sequence $\xi_{n}=\left\{\left|T_{n}\right|^{\theta_{n}},\left|U_{n}\right|^{\theta_{n}}\right\}$ for all values of $\alpha$ and $\beta$. This is presented in $\S 4$. The description of the set $K$ is achieved from Lemmas 3 to 9 of $\S 3$. In $\S 2$, some planar sets and some special integer sequences are defined.

2. Notations and definitions. Define the following sets in the two dimensional plane:

$$
\begin{aligned}
& K_{1}=\left\{\left(e^{a / \alpha}, e^{b / \alpha}\right) ; 0 \leqslant a, b \leqslant 1, a+b \leqslant 1\right\}, \\
& K_{2}=\left\{\left(e^{a / \alpha}, e^{-b}\right) ; 0 \leqslant a \leqslant 1, b \geqslant 0, a+(1-\alpha) b \leqslant 1\right\}, \\
& K_{3}=\left\{\left(e^{-a}, e^{b / \alpha}\right) ; a \geqslant 0,0 \leqslant b \leqslant 1, b+(1-\alpha) a \leqslant 1\right\}, \\
& K_{4}=\left\{\left(e^{-a}, e^{-b}\right) ; a, b \geqslant 0, a+(1-\alpha) b<1\right\} \text { if } 0<\alpha<1 \text {, } \\
& =\left\{\left(e^{-a}, e^{-b}\right) ; 0 \leqslant a \leqslant(2-\alpha)^{-1}, b \geqslant 0, a+(1-\alpha) b<1\right\} \\
& \text { if } 1<\alpha<2 \text {, } \\
& K_{5}=\left\{\left(e^{-a}, e^{-b}\right), a, b \geqslant 0, b+(1-\alpha) a<1\right\} \text { if } 0<\alpha<1 \text {, } \\
& =\left\{\left(e^{-a}, e^{-b}\right), a \geqslant 0,0<b \leqslant(2-\alpha)^{-1}, b+(1-\alpha) a<1\right\} \\
& \text { if } 1 \leqslant \alpha<2 \text {. }
\end{aligned}
$$

Throughout the paper i.o. will stand for infinitely often: $[u]$ will stand for the largest integer $\leqslant u$; and $n_{r}\left(=n_{r}(a, b) ; a, b \geqslant 0\right)$ and $m_{r}$, respectively, will stand for the integer sequences [exp $r^{(a+b)^{-1}}$ ] and [exp $r$ ]. Also $R, C(>0)$ will denote absolute constants ( $R$ an integer), not necessarily the same at each occurrence.

We say that $(x, y)$ is a limit point of $\left(\xi_{n}\right)$ if for every $\delta>0, P\left(\xi_{n} \in N_{\delta}\right.$ i.o.) $=1$ where $N_{\delta}=(x-\delta, x+\delta) \times(y-\delta, y+\delta)$.

3. LEMMA 1. Let $\left(D_{n}\right)$ be a sequence of events in a probability space. If

$$
\text { (i) } \sum_{n=1}^{\infty} P\left(D_{n}\right)=\infty
$$

and

$$
\text { (ii) } \liminf _{n \rightarrow \infty} \frac{\sum_{r=1}^{n} \sum_{s=1}^{n} P\left(D_{r} \cap D_{s}\right)}{\left(\sum_{r=1}^{n} P\left(D_{r}\right)\right)^{2}} \leqslant C,
$$

then $P\left(D_{r}\right.$ i.o. $) \geqslant C^{-1}$.

For proof see [8, p. 317]. 
Lemma 2. Let $S_{1}, S_{2}, \ldots, S_{n}$ be successive sums of independent random variables and let $a_{1}, a_{2}, \ldots, a_{n}$ be a sequence of real constants. Further, let $b$ $(>0)$ and $\alpha$ be constants such that

$$
\sup _{m<j<n} P\left(\left|\left(S_{n}-a_{n}\right)-\left(S_{j}-a_{j}\right)\right|>b\right)=\alpha<1 ;
$$

then

$$
\begin{array}{r}
P\left(\sup _{m<j<n}\left|S_{j}-a_{j}\right|>2 b\right) \leqslant(1-\alpha)^{-1} P\left(\left|S_{n}-a_{n}\right|>b\right), \\
P\left(\inf _{m<j<n}\left|S_{j}-a_{j}\right|<b\right) \leqslant(1-\alpha)^{-1} P\left(\left|S_{n}-a_{n}\right|<2 b\right), \\
m=1,2,3, \ldots, n .
\end{array}
$$

The proof follows on the lines of [1, Lemma 3.21, p. 45] and, hence, is omitted.

Below in Lemmas 3 to 9 , we assume that $\alpha \neq 1$. When $\alpha=1$, nonzero subtracting constants $a_{n}$ 's are present. The lemmas are again true, but the proofs require slightly modified arguments and are omitted.

LeMmA 3. For all $a, b \geqslant 0$ with $a+b \geqslant 1$ and for every $\varepsilon>0$,

$$
P\left(\left|T_{n}\right|^{\theta_{n}}>e^{(a+\varepsilon) / \alpha},\left|U_{n}\right|^{\theta_{n}}>e^{(b+e) / \alpha} \text { i.o. }\right)=0 .
$$

Proof. Define the events $A_{n}, B_{r}$ and $C_{r}$ through

$$
\begin{aligned}
& A_{n}=\left(\left|T_{n}\right|>(\log n)^{(a+\varepsilon) / \alpha},\left|U_{n}\right|>(\log n)^{(b+e) / \alpha}\right), \\
& B_{r}=\left(\left|n^{1 / \alpha} T_{n}\right|>n_{r}^{1 / \alpha}\left(\log n_{r}\right)^{(a+e) / \alpha},\left|n^{1 / \alpha} U_{n}\right|>n_{r}^{1 / \alpha}\left(\log n_{r}\right)^{(b+e) / \alpha}\right. \\
&\left.\quad \text { for at least one } n \text { in } n_{r} \leqslant n<n_{r+1}\right), \\
& C_{r}=\left(\left|n_{r+1}^{1 / \alpha} T_{n_{r+1}}\right|>\frac{n_{r}^{1 / \alpha}}{2}\left(\log n_{r}\right)^{(a+\varepsilon) / \alpha},\left|n_{r+1}^{1 / \alpha} U_{n_{r+1}}\right|>\frac{n_{r}^{1 / \alpha}}{2}\left(\log n_{r}\right)^{(b+e) / \alpha}\right) .
\end{aligned}
$$

Observe that

$$
\text { (i) } P\left(A_{n} \text { i.o. }\right) \leqslant P\left(B_{r} \text { i.o. }\right) \text {, (ii) } P\left(C_{r}\right) \leqslant C r^{-(1+\varepsilon)}
$$

for all $r>R$ and

$$
P\left(\left|n_{r+1}^{1 / \alpha} T_{n_{r+1}}-n^{1 / \alpha} T_{n}\right|>\frac{n_{r}^{1 / \alpha}}{2}\left(\log n_{r}\right)^{(a+e) / \alpha}\right)
$$

(iii) $\quad<P\left(\left|\left(n_{r+1}-n\right)^{-1 / \alpha}\left(n_{r+1}^{1 / \alpha} T_{n_{r+1}}-n^{1 / \alpha} T_{n}\right)\right|>\frac{n_{r}^{1 / \alpha}}{2}\left(\log n_{r}\right)^{(a+\varepsilon) / \alpha}\right)$

$$
<P\left(\left|X_{1}\right|>\frac{n_{r}^{1 / \alpha}}{2}\left(n_{r+1}-n_{r}\right)^{-1 / \alpha}\left(\log n_{r}\right)^{(a+\varepsilon) / \alpha}\right)<\frac{1}{2}
$$


for all $r>R$ and for all $n$ in $n_{r} \leqslant n<n_{r+1}$. Similar to (iii) one may obtain for all $r>R$ and for all $n$ in $n_{r} \leqslant n<n_{r+1}$,

$$
P\left(\left|n_{r+1}^{1 / \alpha} U_{n_{r+1}}-n^{1 / \alpha} U_{n}\right|>\frac{n_{r}^{1 / \alpha}}{2}\left(\log n_{r}\right)^{(b+e) / \alpha}\right)<\frac{1}{2} .
$$

Now from (iii), (iv) and Lemma 2, $P\left(B_{r}\right)<4 P\left(C_{r}\right)$ for all $r \geqslant R$, and hence, $\sum_{r=1}^{\infty} P\left(B_{r}\right)<\infty$. An appeal to the Borel-Cantelli lemma completes the proof.

LEMMA 4. For every $\varepsilon>0, P\left(\left|T_{n}\right|^{\theta_{n}}>e^{(1+\varepsilon) / \alpha} i . o.\right)=0$.

Proof. The proof is exactly similar to Lemma 3 by considering the events $\left(\left|T_{n}\right|>(\log n)^{(1+\varepsilon) / \alpha}\right),\left(\sup _{m_{r}<n<m_{r+1}}\left|n^{1 / \alpha} T_{n}\right|>m_{r}^{1 / \alpha}\left(\log m_{r}\right)^{(1+\varepsilon) / \alpha}\right)$ and $\left(\left|m_{r+1}^{1 / \alpha} T_{m_{r+1}}\right|>\left(m_{r}^{1 / \alpha} / 2\right)\left(\log m_{r}\right)^{(1+\varepsilon) / \alpha}\right)$, respectively, in place of $A_{n}, B_{r}$ and $C_{r}$.

LeMma 5. If $0<\alpha<1$ and if $\beta= \pm 1$, then for every $\varepsilon>0, P\left(\left|T_{n}\right|^{\theta_{n}}<e^{-e}\right.$ i.o.) $=0$.

Proof. Define

$$
V_{n}=\left(\left|T_{n}\right|<(\log n)^{-\varepsilon}\right)
$$

and

$$
W_{r}=\left(\inf _{m_{r}<n<m_{r+1}}\left|n^{1 / \alpha} T_{n}\right|<m_{r+1}^{1 / \alpha}\left(\log m_{r}\right)^{-\varepsilon}\right) .
$$

Then

$$
P\left(V_{n} \text { i.o. }\right) \leqslant P\left(W_{r} \text { i.o. }\right)=P\left(\left|m_{r}^{1 / \alpha} T_{m_{r}}\right|<m_{r+1}^{1 / \alpha}\left(\log m_{r}\right)^{-e} \text { i.o. }\right) \text {. }
$$

Recall $e^{x^{-a}} G_{\alpha}(x) \rightarrow 0$ as $x \rightarrow 0$ for the distribution function $G_{\alpha}(x)$ of a positive stable random variable [4, p. 424]. Therefore

$$
P\left(\left|m_{r}^{1 / \alpha} T_{m_{r}}\right|<m_{r+1}^{1 / \alpha}\left(\log m_{r}\right)^{-\varepsilon}\right)<c\left(\exp -r^{\varepsilon / \alpha}\right)
$$

for all $r>R$. Hence $\sum_{r=1}^{\infty} P\left(W_{r}\right)<\infty$ and the lemma is proved.

LEMMA 6. For every $\varepsilon>0, P\left(\left|T_{n}\right|^{\theta_{n}}<e^{-(a+\varepsilon)},\left|U_{n}\right|_{n}<e^{-(b+\varepsilon)}\right.$ i.o. $)=0$. Here $a, b$ satisfy (i) $a, b>0$ and (ii) $a+(1-\alpha) b>1$ and $b+(1-\alpha) a>1$.

Proof. Define

$$
\begin{aligned}
A_{n}=\left(\left|T_{n}\right|<(\log n)^{-(a+\varepsilon)},\left|U_{n}\right|<(\log n)^{-(b+\varepsilon)}\right) & \\
B_{r}=\left(\left|n^{1 / \alpha} T_{n}\right|<n_{r+1}^{1 / \alpha}\left(\log n_{r}\right)^{-(a+\varepsilon)},\right. & \left|n^{1 / \alpha} U_{n}\right|<n_{r+1}^{1 / \alpha}(\log n)^{-(b+e)} \\
& \text { for at least one } \left.n \text { in } n_{r}<n<n_{r+1}\right)
\end{aligned}
$$

and

$$
C_{r}=\left(\left|T_{n_{r+1}}\right|<2\left(\log n_{r}\right)^{-(a+\varepsilon)},\left|U_{n_{r+1}}\right|<2\left(\log n_{r}\right)^{-(b+\varepsilon)}\right)
$$


As in Lemma 3 we see that for all $r>R$ and for all $n$ in $n_{r}<n<n_{r+1}$,

$$
P\left(\left|n_{r+1}^{1 / \alpha} T_{n_{r+1}}-n^{1 / \alpha} T_{n}\right|>n_{r+1}^{1 / \alpha}\left(\log n_{r}\right)^{-(a+\varepsilon)}\right)<\frac{1}{2}
$$

and

$$
P\left(\left|n_{r+1}^{1 / \alpha} U_{n_{r+1}}-n^{1 / \alpha} U_{n}\right|>n_{r+1}^{1 / \alpha}\left(\log n_{r}\right)^{-(b+e)}\right)<\frac{1}{2} .
$$

In claiming these, use is made of the fact that

$$
n_{r+1}^{1 / \alpha}\left(n_{r+1}-n_{r}\right)^{-1 / \alpha}\left(\log n_{r}\right)^{-(\lambda+\varepsilon)} \rightarrow \infty \quad \text { as } r \rightarrow \infty,
$$

if $\lambda=a$ and $a+(1-\alpha) b>1$ or if $\lambda=b$ and $b+(1-\alpha) a>1$. Further, as the density of $X_{1}$ exists, $P\left(C_{r}\right) \leqslant C r^{-(1+e)}$ for all $r \geqslant R$. An application of Lemma 2 now implies that $\sum_{r=1}^{\infty} P\left(B_{r}\right)<\infty$. Hence by the Borel-Cantelli lemma and by the fact that $P\left(A_{n}\right.$ i.o. $) \leqslant P\left(B_{r}\right.$ i.o. $)$, the proof is complete.

LEMMA 7. For any $\varepsilon>0$ and for $0<\alpha<1$,

$$
P\left(\left|T_{n}\right|^{\theta_{n}}>e^{(a+\varepsilon) / \alpha},\left|U_{n}\right|^{\theta_{n}}<e^{-(b+\varepsilon)} \text { i.o. }\right)=0
$$

when $a, b>0$ satisfy $a+(1-\alpha) b>1$,

$$
P\left(\left|T_{n}\right|^{\theta_{n}}<e^{-(a+\varepsilon)},\left|U_{n}\right|^{\theta_{n}}>e^{(b+\varepsilon) / \alpha} \text { i.o. }\right)=0
$$

when $a, b \geqslant 0$ satisfy $b+(1-\alpha) a>1$.

Proof is on the lines of Lemmas 3 and 6 . The details are omitted.

LEMMA 8. For every $\varepsilon>0$ and for all $a, b \geqslant 0$

(i) $\quad P\left(\left|T_{n_{r}}\right|^{\theta_{n_{r}}}>e^{(a+\varepsilon) / \alpha},\left|U_{n_{r}}\right|^{\theta_{n_{r}}}>e^{b / \alpha}\right.$ i.o. $)=0$,

(ii) $P\left(\left|T_{n}\right|^{\theta_{n_{r}}}>e^{a / \alpha},\left|U_{n}\right|^{\theta_{n_{r}}}>e^{(b+e) / \alpha}\right.$ i.o. $)=0$,

(iii) $P\left(\left|T_{n_{r}}\right|^{\theta_{n_{r}}}>e^{(a+e) / \alpha},\left|U_{n_{r}}\right|^{\theta_{n_{r}}}<e^{-b}\right.$ i.o. $)=0$,

(iv) $P\left(\left|T_{n_{r}}\right|^{\theta_{n_{r}}}>e^{a / \alpha},\left|U_{n_{r}}\right|^{\theta_{n_{r}}}<e^{-(b+\varepsilon)}\right.$ i.o. $)=0$,

(v) $P\left(\left|T_{n_{r}}\right|^{\theta_{n_{r}}}<e^{-(a+\varepsilon)},\left|U_{n_{r}}\right|^{\theta_{n_{r}}}>e^{b / \alpha}\right.$ i.o. $)=0$,

(vi) $P\left(\left|T_{n_{r}}\right|^{\theta_{n_{r}}}<e^{-a},\left|U_{n_{r}}\right|^{\theta_{n_{r}}}>e^{(b+\varepsilon) / \alpha}\right.$ i.o. $)=0$,

(vii) $\quad P\left(\left|T_{n_{r}}\right|^{\theta_{n_{r}}}<e^{-(a+\varepsilon)},\left|U_{n_{r}}\right|^{\theta_{n_{r}}}<e^{-b}\right.$ i.o. $)=0$,

(viii) $P\left(\left|T_{n_{r}}\right|^{\theta_{n_{r}}}<e^{-a},\left|U_{n_{r}}\right|^{\theta_{n_{r}}}<e^{-(b+\varepsilon)}\right.$ i.o. $)=0$.

Proof. A direct application of the Borel-Cantelli lemma yields (i) through (viii).

Lemmas 3 and 7 above help us identify the points which are not limit points of $\left(\xi_{n}\right)$, while Lemma 8 identifies the points which are not the limit 
points of the subsequence $\left(\xi_{n_{n}}\right)$ for specific values of $a, b$ of interest. Lemma 9 below helps identify the points which are the limit points of $\left(\xi_{n}\right)$. This identification is done by showing that with positive probability the sequence $\left(\xi_{n}\right)$ lies i.o. in every neighbourhood of a point specified by $a$ and $b$ and by appealing to the Hewitt-Savage zero-one law.

Lemma 9.

$$
P\left(\left|T_{n_{r}}\right|^{\theta_{n_{r}}}>e^{a / \alpha},\left|U_{n_{r}}\right|^{\theta_{n_{r}}}>e^{b / \alpha} \text { i.o. }\right)=1
$$

$$
\text { if } a, b>0 \text { and } a+b<1 \text {. }
$$

$$
\begin{aligned}
& \text { (ii) } P\left(\left|T_{n_{1}}\right|^{\theta_{n_{r}}}>e^{a / \alpha},\left|U_{n_{n}}\right|^{\theta_{n_{r}}}<e^{-b} \text { i.o. }\right)=1 \\
& \text { if } 0 \leqslant a<1, b>0 \text { and } a+(1-\alpha) b<1 \text {. } \\
& \text { (3.3) (iii) } P\left(\left|T_{n_{n}}\right|^{\theta_{n_{r}}}<e^{-a},\left|U_{n_{l}}\right|^{\theta_{n_{r}}}>e^{b / \alpha} \text { i.o. }\right)=1 \\
& \text { if } a \geqslant 0,0<b<1 \text { and } b+(1-\alpha) a \leqslant 1 \text {. } \\
& \text { (iv) } P\left(\left|T_{n_{r}}\right|^{\theta_{n_{r}}}<e^{-a},\left|U_{n_{r}}\right|^{\theta_{n_{r}}}<e^{-b} \text { i.o. }\right)=1 \\
& \text { if } a, b>0 \text { for } 0<\alpha<1,0<a<(2-\alpha)^{-1} \text {, } \\
& b \geqslant 0 \text { for } 1<\alpha<2 \text { and } a+(1-\alpha) b<1 \text {. }
\end{aligned}
$$

$$
\begin{aligned}
\text { (v) } P\left(\left|T_{n_{r}}\right|^{\theta_{n_{r}}}<e^{-a},\left|U_{n_{r}}\right|^{\theta_{n_{r}}}<e^{-b} \text { i.o. }\right)=1 \\
\text { if } a, b>0 \text { for } 0<\alpha<1, a \geqslant 0,0<b<(2-\alpha)^{-1} \\
\text { for } 1 \leqslant \alpha<2 \text { and } b+(1-\alpha) a<1 .
\end{aligned}
$$

Proof. We prove (4.3) below. Similar proofs for (1.3) and (2.3) can be given. Since $T_{n_{r}}$ and $U_{n_{r}}$ are interchangeable, (3.3) and (5.3) then follow by reasons of symmetry. The details are omitted.

Define

$$
D_{r}=\left(\left|T_{n_{1}}\right|<\left(\log n_{r}\right)^{-a},\left|U_{n_{1}}\right|<\left(\log n_{r}\right)^{-b}\right) .
$$

In view of our earlier remarks, to establish (4.3) it is sufficient to show that $P\left(D_{r}\right.$ i.o. $)>0$.

Observe that $\sum_{r=1}^{n} P\left(D_{r}\right) \sim c(\log n)$ and, consequently, as $n \rightarrow \infty$,

$$
\left(\sum_{r=1}^{n} P\left(D_{r}\right)\right)^{-2} \sum_{r=1}^{n} \sum_{s=1}^{n} P\left(D_{r} \cap D_{s}\right)
$$

is asymptotically

$$
2\left(\sum_{r=1}^{n} P\left(D_{r}\right)\right)^{-2} \sum_{r=1}^{n-1} \sum_{s=r+1}^{n} P\left(D_{r} \cap D_{s}\right) .
$$

Below we find a suitable upper bound for 


$$
\sum_{r=1}^{n-1} \sum_{s=r+1}^{n} P\left(D_{r} \cap D_{s}\right)\left(\sum_{r=1}^{n} P\left(D_{r}\right)\right)^{-2} \text {. }
$$

$$
\begin{aligned}
& \text { If }(a+b) \leqslant 1 \text {, then } n_{r} / n_{s}<e^{-1}+\delta<1 \text { for some } \delta>0 \text { and, hence, } \\
& \begin{aligned}
& P\left(\left|T_{n_{r}}\right|<\left(\log n_{r}\right)^{-a},\left|T_{n_{s}}\right|<\left(\log n_{s}\right)^{-a}\right) \\
& \quad<P\left[\left|T_{n_{r}}\right|<\left(\log n_{r}\right)^{-a},\left|\frac{S_{1, n_{s}}-S_{1, n_{r}}}{\left(n_{s}-n_{r}\right)^{1 / \alpha}}\right|<c\left(\log n_{s}\right)^{-a}\right] \\
&<P\left(\left|T_{n_{r}}\right|<\left(\log n_{r}\right)^{-a}\right) P\left(\left|X_{1}\right|<c\left(\log n_{s}\right)^{-a}\right) \text { for all } r \geqslant R \\
&<c P\left(\left|T_{n_{r}}\right|<\left(\log n_{r}\right)^{-a}\right) P\left(\left|T_{n_{s}}\right|<\left(\log n_{s}\right)^{-a}\right) \text { for all } r>R .
\end{aligned}
\end{aligned}
$$

Repeating the steps for $\left(U_{n}\right)$ we can write $P\left(D_{r} \cap D_{s}\right)<c P\left(D_{r}\right) P\left(D_{s}\right)$ for $r \geqslant R$. Hence (1) and (2) readily hold.

Suppose now $(a+b)>1$; two cases arise.

Case $1 .(a+b)>1$ but $a<1$.

Define $q=(a+b-1)(a+b)^{-1}$ and $\varphi=\min \left(n,\left[r+r^{q}\right]\right)$ and write

$$
\sum_{r=1}^{n-1} \sum_{s=r+1}^{n} P\left(D_{r} \cap D_{s}\right) \leqslant \sigma_{1}+\sigma_{2}
$$

where

$$
\sigma_{1}=\sum_{r=1}^{n-1} \sum_{s=r+1}^{\left[r+r^{q}\right]} P\left(D_{r} \cap D_{s}\right) \text { and } \sigma_{2}=\sum_{r=1}^{n-1} \sum_{s=\varphi}^{n} P\left(D_{r} \cap D_{s}\right) .
$$

For $(r+1) \leqslant s \leqslant\left[r+r^{q}\right]$ and for all $r>R$,

$$
P\left(\left|U_{n_{r}}\right|<\left(\log n_{r}\right)^{-b},\left|U_{n_{s}}\right|<\left(\log n_{s}\right)^{-b}\right)<c\left(1-n_{r} / n_{s}\right)^{-1 / a}(r s)^{-b /(a+b)} \text {. }
$$

Hence

$$
\begin{aligned}
P\left(D_{r} \cap D_{s}\right) & \leqslant c\left(1-n_{r} / n_{s}\right)^{-1 / \alpha}(r s)^{-b /(a+b)} P\left(\left|T_{n_{r}}\right|<\left(\log n_{r}\right)^{-a}\right) \\
& \leqslant c\left(1-n_{r} / n_{s}\right)^{-1 / \alpha} r^{-1} s^{-b /(a+b)} .
\end{aligned}
$$

Easy calculations lead to $\left(1-n_{r} / n_{s}\right)^{-1 / \alpha} \leqslant c r^{q / \alpha}(s-r)^{-1 / \alpha}$. Thus

$$
\sigma_{1} \leqslant \text { Const }+c \sum_{r=1}^{n-1} r^{(q / \alpha-(a+2 b) /(a+b))} \sum_{k=1}^{r q} k^{-1 / \alpha} \leqslant c(\log n) .
$$

When $s>\left[r+r^{q}\right]$ it is trivial to see that $n_{r} / n_{s}<e^{-1}+\delta<1$ for some $\delta>0$. Consequently for all $r>R, P\left(D_{r} \cap D_{s}\right) \leqslant c P\left(D_{r}\right) P\left(D_{s}\right)$. Since 
(4)

$$
\begin{gathered}
\sum_{r=1}^{R} \sum_{s=\varphi}^{n} P\left(D_{r} \cap D_{s}\right) \leqslant R \sum_{s=1}^{n} P\left(D_{s}\right) \leqslant c(\log n), \\
\sigma_{2} \leqslant c \log n+c \sum_{r=R}^{n} \sum_{s=1}^{n} P\left(D_{r}\right) P\left(D_{s}\right) \leqslant c(\log n)^{2} .
\end{gathered}
$$

(3), (4) and Lemma 1 now guarantee that $P\left(D_{r}\right.$ i.o. $)>0$.

When $0<\alpha<1$, the proof of (4.3) is complete. When $\alpha>1$, there are other values of $a, b$ which are discussed in Case 2 .

Case 2. $(a+b)>1$ and $1<a<(2-\alpha)^{-1} ; 1<\alpha<2$. Define

$$
p=(1-a+(\alpha-1) b) /(a+b)(\alpha-1)
$$

and note that $p>0$. Take $q$ and $\varphi$ as given in Case 1 and observe that $p<q$. Now write

$$
\sum_{r=1}^{n-1} \sum_{s=r+1}^{n} P\left(D_{r} \cap D_{s}\right)<\Delta_{1}+\Delta_{2}+\Delta_{3}
$$

where

$$
\begin{aligned}
& \Delta_{1}=\sum_{r=1}^{n-1} \sum_{s=r+1}^{\left[r+r^{p]}\right.} P\left(D_{r} \cap D_{s}\right), \\
& \Delta_{2}=\sum_{r=1}^{n-1} \sum_{s=\left[r+r^{p]}\right.}^{\left[r+r^{q]}\right]} P\left(D_{r} \cap D_{s}\right) \text { and } \\
& \Delta_{3}=\sum_{r=1}^{n} \sum_{s=\varphi}^{n} P\left(D_{r} \cap D_{s}\right) .
\end{aligned}
$$

If $s \in\left(r,\left[r+r^{p}\right]\right)$ and if $r \geqslant R$, then

$$
P\left(D_{r} \cap D_{s}\right)<c\left(1-n_{r} / n_{s}\right)^{-1 / \alpha} r^{-1} s^{-b /(a+b)},
$$

and proceeding as in Case 1 one gets

$$
\Delta_{1} \leqslant c(\log n) \text {. }
$$

If $s \in\left(\left[r+r^{p}\right],\left[r+r^{q}\right]\right)$ and if $r \geqslant R$, then

$$
P\left(\left|T_{n_{1}}\right|<\left(\log n_{r}\right)^{-a},\left|T_{n_{s}}\right|<\left(\log n_{s}\right)^{-a}\right)<c\left(1-n_{r} / n_{s}\right)^{-1 / \alpha}(r s)^{-a /(a+b)}
$$

and

$$
\begin{aligned}
P\left(\left|U_{n_{r}}\right|<\right. & \left.\left(\log n_{r}\right)^{-b},\left|U_{n_{s}}\right|<\left(\log n_{s}\right)^{-b}\right) \\
& <c\left(1-n_{r} / n_{s}\right)^{-1 / \alpha}(r s)^{-b /(a+b)} .
\end{aligned}
$$

Hence

$$
\Delta_{2} \leqslant c \log n+c \sum_{r=R}^{n} r^{2(q-p-\alpha) / \alpha} \sum_{s=\left[r+r^{p}\right]}^{\left[r+r^{q}\right]} K^{-2 / \alpha} \leqslant c(\log n) .
$$


For $s>\varphi$, by arguments similar to the ones given in Case 1 we get

$$
\Delta_{3}<c(\log n)^{2} \text {. }
$$

From (5), (6) and (7) we conclude that a $c$ exists such that (2) holds and, consequently, $P\left(D_{r}\right.$ i.o. $)>0$. The proof is now completed.

4. THEOREM. The set $K$ of all the limit points of the sequence $\left(\xi_{n}\right)$ is given by

$$
K= \begin{cases}K_{1} & \text { if } 0<\alpha<1 \text { and } \beta= \pm 1 \\ \bigcup_{j=1}^{5} K_{j} & \text { if } 0<\alpha<1,|\beta|<1 \text { or } 1<\alpha<2 .\end{cases}
$$

Proof. When $0<\alpha<1$ and $|\beta|=1$, Lemmas 3 and 5 establish that the limit points of $\left(\xi_{n}\right)$ must be in $K\left(=k_{1}\right)$ only; (1.1), (1.2) and (1.3) establish that every point in $K$ is a limit point.

That no point outside $K$ is a limit point of $\left(\xi_{n}\right)$ follows from Lemmas 3, 4, 6 and 7 in the case $0<\alpha<1,|\beta|<1$ and from Lemmas 3,4 and 6 in the case $1<\alpha<2$. In both cases, that every point in $K_{j}$ is a limit point of $\left(\xi_{n}\right)$ follows from $(j .1),(j .2)$ and $(j .3)$ for $j=1,2,3,4$, and from (4.1), (4.2) and (5.3) for $j=5$.

ReMARK 1. When $\xi_{n}$ has $p(>3)$ components the proof is on similar lines. We describe below the limit set $K_{p}(\alpha)$ in $p$ dimensions.

Write

$$
\xi_{n}(m)=\left(\left|T_{1, n}\right|^{\theta_{n}},\left|T_{2, n}\right|^{\theta_{n}} \ldots\left|T_{m, n}\right|^{\theta_{n}}\right),
$$

$m=1,2,3, \ldots$, where $T_{j, n}=n^{-1 / \alpha}\left(S_{j, n}-a_{n}\right)$ and $S_{j, n}, j=1,2, \ldots, m$, are independent copies of $S_{n}$. Let $C_{m}(\alpha)$ be the $m$-dimensional cube $\left[0, e^{1 / \alpha}\right]^{m}$.

For an arbitrary point $X_{p}=\left(x_{1}, \ldots, x_{p}\right)$ in $C_{p}(\alpha)$ let $q=q\left(X_{p}\right)$ denote the number of components with values in $[0,1) ; r=r\left(X_{p}\right)$ be the minimum of these components if $q \geqslant 1$ and be equal to 1 if $q=0 ; s=s\left(X_{p}\right)$ be the maximum of these components if $q>1$ and be equal to 1 if $q=0$.

Define the functions

$$
\begin{aligned}
& A(x)= \begin{cases}1 & \text { if } 0<x<1, \\
x^{\alpha} & \text { if } 1<x<e^{1 / \alpha}\end{cases} \\
& B(x)= \begin{cases}x^{-1} & \text { if } 0<x<1, \\
1 & \text { if } 1<x<e^{1 / \alpha}\end{cases}
\end{aligned}
$$

Let $u=e^{-1} \Pi_{j=1}^{p} A\left(x_{j}\right)$ and $v=\prod_{j=1}^{p} B\left(x_{j}\right)$.

For $p>2$, a point $X_{p+1}=\left(x_{1}, x_{2}, \ldots, x_{p+1}\right)$ is a limit point of $\xi_{n}(p+1)$, i.e. $X_{p+1} \in K_{p+1}(\alpha)$, if and only if

(i) $X_{p+1} \in C_{p+1}(\alpha)$,

(ii) $X_{p} \in K_{p}(\alpha), x_{p+1} \in K_{1}(\alpha)$, and

(iii) either $0<\alpha<1$ and 


$$
\begin{aligned}
& 0<\min \left\{u v r^{\alpha},(u v)^{1 /(1-\alpha)}\right\} \leqslant x_{p+1}<1 \text {, or } \\
& 0<\alpha<1 \text { and } \max \left\{e^{-1}, u v r^{\alpha}\right\} \leqslant x_{p+1}^{-\alpha} \leqslant 1 \text {, or } \\
& 1 \leqslant \alpha<2, q=0 \text { and } 0 \leqslant x_{p+1}<1 \text {, or } \\
& 1 \leqslant \alpha<2, q \geqslant 1,0 \leqslant x_{p+1}<1 \text { and } \min \left(r, x_{p+1}\right)>u^{1 /(q+1-\alpha)} \text {, or } \\
& 1<\alpha<2 \text { and } \max \left\{e^{-1}, u s^{-(q-\alpha)}\right\} \leqslant x_{p+1}^{-\alpha} \leqslant 1 .
\end{aligned}
$$

REMARK 2. When the summands are standard normal variables, minor modifications of our steps establish that every point in the unit square $[0,1]^{2}$ is a limit point of $\left(\xi_{n}\right)$. That no point outside this square is a limit point of $\left(\xi_{n}\right)$ is a consequence of the classical law of the iterated logarithm [5]. When $\xi_{n}$ has $p(\geqslant 3)$ components, the set $K_{p}(2)$ of the limit points can, on similar lines, be proved to be $[0,1]^{p}-\left[0, e^{-1 /(p-2)}\right]^{p}$. Thus for $p \geqslant 3$, the limit set is not the cube. For a different sequence $\left(\xi_{n}\right)$, LePage [6] has obtained the limit set to be the unit ball in space of $p$-dimensions.

REMARK 3. One may consider component sequences (sign $\left.T_{n}\right)\left|T_{n}\right|^{\theta_{n}}$ and arrive at all the limit points following closely our steps for the multidimensional situation. In case $\alpha \geqslant 1$ and $\beta= \pm 1$ use will have to be made of the fact that the appropriate tail of the distribution tends to zero exponentially fast.

ACKNOWLEDGEMENT. The authors thank the referee of an earlier version of this paper for his valuable comments and for bringing to their notice reference [7].

\section{REFERENCES}

1. L. Breiman, Probability, Addison-Wesley, Reading, Mass., 1968. MR 37 \#4841.

2. J. Chover, $A$ law of the iterated logarithm for stable summands, Proc. Amer. Math. Soc. 17 (1966), 441-443. MR 32 \#6523.

3. K. L. Chung and W. H. J. Fuchs, On the distribution of values of sums of random variables, Mem. Amer. Math. Soc. No. 6 (1951), 12 pp. MR 12, 722.

4. W. Feller, An irtroduction to probability theory and its applications, Vol. 2, Wiley, New York, 1966. MR 35 \# 1048.

5. P. Hartman and A. Wintner, On the law of the iterated logarithm, Amer. J. Math. 63 (1941), 169-176. MR 2, 228.

6. R. D. LePage, Log log law for gaussian processes, Z. Wahrscheinlichkeitstheorie und Verw. Gebiete 25 (1972/73), 103-108. MR 48 \# 9789.

7. J. L. Mijnheer, Sample path properties of stable processes, Math. Centre Tracts, no. 59, Mathematische Centrum, Amsterdam, 1975. MR 51 \# 7008.

8. F. Spitzer, Principles of random walk, Van Nostrand, Princeton, N.J., 1964. MR 30 \#1521.

Department of Statistics, University of Mysore, Manasagangotri, Mysore 570006, INDIA 